\title{
Reconnexion magnétique entre le vent solaire et la magnétosphère terrestre : la mission MMS
}

Laurence Rezeau (laurence.rezeau@lpp.polytechnique.fr) et Gérard Belmont

Laboratoire de Physique des Plasmas (CNRS, École polytechnique, UPMC, Univ. Paris-Sud, OBSPM, Université Paris-Saclay, Sorbonne Universités, PSL Research University) LPP, École polytechnique, 91128 Palaiseau Cedex

Dans le vent solaire, plasma

et champ magnétique se déplacent ensemble à grande échelle.

L'interface avec la magnétosphère terrestre est une frontière fine, la magnétopause, où il peut exister des échelles suffisamment petites où les deux mouvements se dissocient. Il en résulte un phénomène nommé

" reconnexion magnétique », au cours duquel le plasma est fortement accéléré le long de la frontière.

La mission MMS a des points forts qui en font le meilleur outil pour étudier ce phénomène : une résolution temporelle des mesures inégalée, et des satellites très proches les uns des autres (environ $10 \mathrm{~km}$, de l'ordre du rayon de Larmor des électrons dans les plasmas rencontrés).

\section{MMS : une mission multisatellite d'étude de la magnétosphère}

Lancée en 2015, la mission MMS est une mission multisatellite de la NASA, dont l'acronyme signifie "Magnetospheric MultiScale". À l'instar de la mission Cluster de l'ESA (lancée en 2000 et toujours en opération) qui l'a précédée, MMS est composée de quatre satellites identiques qui prennent des mesures simultanées, de manière à observer la géométrie et la dynamique des régions traversées. Ces satellites évoluent dans la magnétosphère de la Terre suivant une orbite elliptique excentrique qui leur permet d'aller jusque dans le vent solaire. Ils sont équipés de nombreux instruments qui permettent de faire in situ des mesures des champs et des propriétés des particules.

La magnétosphère est la bulle de plasma magnétisé qui entoure la Terre (fig. 1). Elle est limitée par une frontière extérieure fine (d'environ $500 \mathrm{~km}$ d'épaisseur), la magnétopause, qui la sépare du plasma magnétisé du vent solaire. Ce dernier venant souffler sur la magnétosphère à une vitesse supersonique supérieure à toutes les vitesses de propagation des modes propres dans le plasma, un choc d'étrave existe en amont de la magnétopause, créant une zone intermédiaire entre ces deux frontières appelée "magnétogaine ", où le plasma ralenti est plus dense et plus chaud.

Cette image simple décrit bien la forme stationnaire moyenne de la magnétosphère, mais elle ne reflète pas complètement la réalité. Les observations montrent que ces régions sont dynamiques, que ces frontières s'agitent sans cesse et que la magnétopause n'est pas toujours et partout une frontière étanche. Les ordres de grandeur des principaux paramètres physiques des différents plasmas au voisinage de la Terre sont donnés dans le tableau 1.

\begin{tabular}{|c|c|c|c|}
\hline Région & $\begin{array}{c}\text { Vent solaire } \\
(1 \text { u.a. })\end{array}$ & Magnétogaine & Magnétosphère \\
\hline Densité $\left(\mathrm{cm}^{-3}\right)$ & 3 & 10 & 0,5 \\
$B(\mathrm{nT})$ & 10 & 30 & 40 \\
\hline$T_{\text {électrons }}(\mathrm{eV})$ & 10 & 30 & 100 \\
\hline$T_{\text {ions }}(\mathrm{eV})$ & 10 & 300 & 2000 \\
\hline
\end{tabular}

Tableau 1. Ordres de grandeur des principaux paramètres physiques dans les différentes régions traversées allant du Soleil vers la Terre : densité des plasmas, champ magnétique B, températures des électrons et des ions. Pour le vent solaire, les valeurs sont données pour une distance d'une unité astronomique du Soleil (donc au voisinage de la Terre). Les frontières sont le choc entre le vent solaire et la magnétogaine, puis la magnétopause entre la magnétogaine et la magnétosphère. 


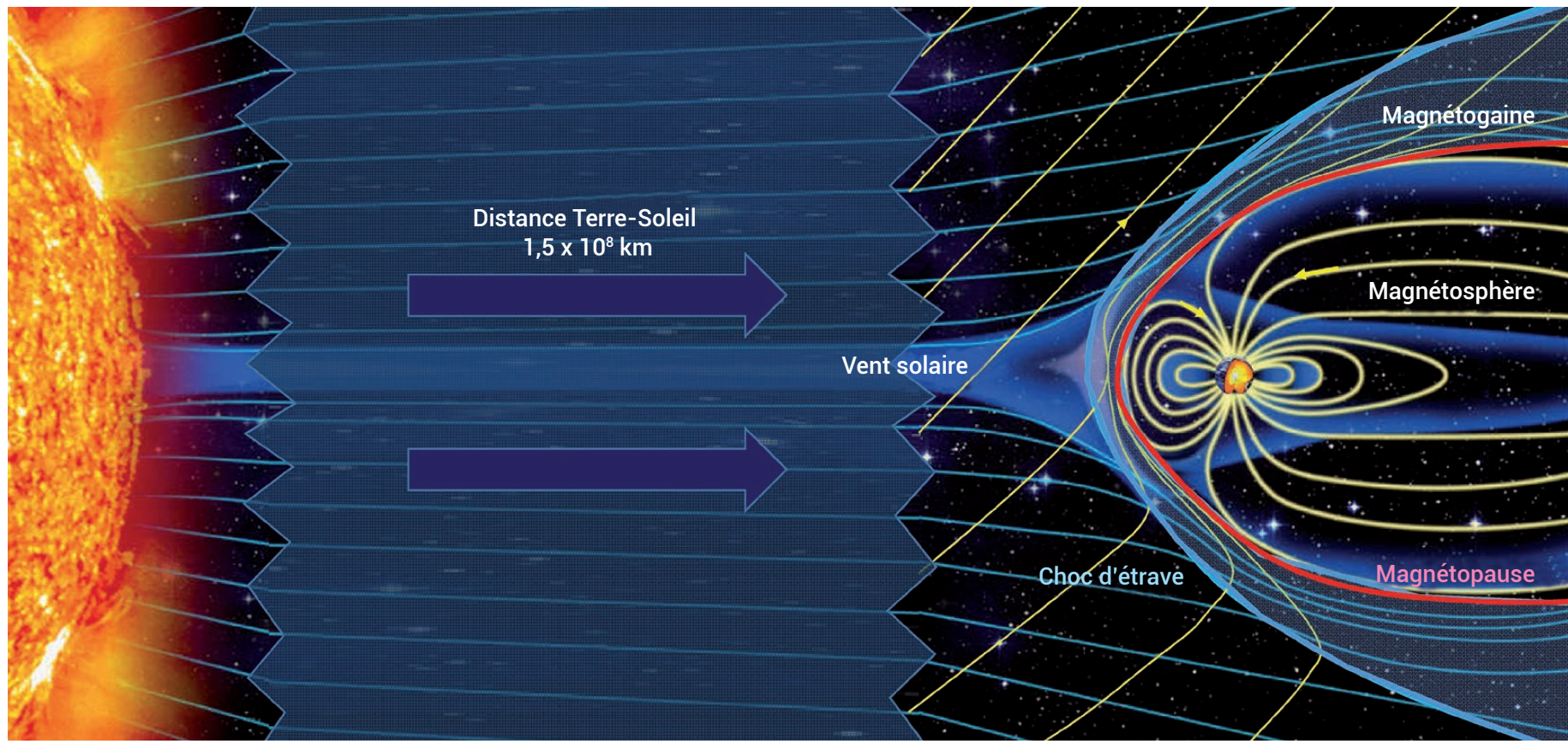

1. Vue d'artiste illustrant l'interaction entre le vent solaire et l'environnement de la Terre (d'après une image de la NASA). Les lignes de champ sont en jaune : celles de la magnétosphère sont connectées à la Terre, celles du vent solaire sont connectées au Soleil (cette connexion n'est pas représentée, compte tenu de la différence d'échelle entre la distance Terre-Soleil et la dimension de la magnétosphère). À l'intérieur de la magnétosphère, on voit que les lignes de champ sont très déformées par rapport au champ d'origine interne seul (quasi dipolaire). Le « nez » de la magnétosphère est à environ 10 rayons terrestres $(60000 \mathrm{~km})$, alors que la « queue magnétosphérique » s'étend à plus de 200 rayons terrestres (au-delà de l'orbite de la Lune). Les différentes frontières sont indiquées par des traits colorés.

Le constat ci-dessus a amené les scientifiques à proposer des missions multisatellites pour pouvoir séparer les effets spatiaux des effets temporels : il est en effet difficile, quand on a un seul point de mesure, de distinguer si une variation dans le temps est due à la traversée d'une structure purement spatiale ou si elle implique un phénomène intrinsèquement temporel. Avec quatre points de mesure, disposés en tétraèdre, on peut surmonter cette difficulté.

La mission Cluster a permis de développer des outils spécifiques à ces mesures en plusieurs points : le "curlometer" (du nom anglais du rotationnel), calcul de la densité de courant à partir de l'équation de Maxwell-Ampère ; et le filtrage en k, estimation de la transformée de Fourier spatiale des ondes, qui permet d'accéder à leur relation de dispersion. Les nombreuses observations faites par Cluster depuis l'an 2000 ont permis d'améliorer la connaissance des grandes régions de la magnétosphère, mais elles ont montré aussi des limites dans la possibilité d'accéder aux petites échelles.

\section{La reconnexion magnétique}

L'objectif central de la mission MMS est l'étude de la reconnexion magnétique dans les frontières de la magnétosphère. Qu'estce que la reconnexion et en quoi les propriétés déjà décrites de la mission permettent-elles des avancées sur la question? Quand on étudie les plasmas spatiaux, on peut émettre l'hypothèse que le champ magnétique est "gelé » dans le plasma aux grandes échelles. Cette propriété découle de l'une des équations de base de la MHD (MagnétoHydroDynamique) : la loi d'Ohm dite idéale. Dans les milieux non collisionnels comme ceux étudiés ici, elle est valable pour les échelles plus grandes que les "longueurs d'inertie " de toutes les espèces $s$ de particules $\left(\delta_{\mathrm{s}}=\mathrm{c} / \omega_{\mathrm{ps}}\right.$, où $\omega_{\mathrm{ps}}$ est la pulsation plasma de l'espèce et $\mathrm{c}$ la vitesse de la lumière). La longueur d'inertie est l'échelle caractéristique du gel du champ magnétique dans le mouvement de chaque espèce : pour les échelles plus grandes, la force électrique est équilibrée par la force de Lorentz; pour les échelles plus petites, elle l'est par la force d'inertie. Pour les électrons, $\delta_{\mathrm{e}}$ vaut typiquement $10 \mathrm{~km}$, ce qui est l'ordre de grandeur de la séparation des satellites MMS. Dans le cas du champ gelé, on peut définir un mouvement des lignes de champ. Les ions et les électrons tournent autour de ces lignes de champ et les suivent donc en moyenne. Cette simple hypothèse de champ gelé permet d'expliquer pourquoi l'essentiel du vent solaire doit contourner la magnétosphère et pourquoi les lignes de champ doivent se " draper " autour d'elle. Cependant, elle se trouve prise en défaut à certains endroits. À l'avant de la magnétosphère par exemple, où le vent solaire comprime fortement le champ magnétique terrestre, des phénomènes à une échelle plus petite que l'échelle MHD peuvent apparaître à la frontière, violant ainsi l'hypothèse du champ gelé. Là où cela se produit, le champ magnétique s'écarte localement de celui qui correspond au mouvement idéal des lignes de champ. Il s'ensuit des changements brutaux des " connexions magnétiques" (fig. 2). On dit 

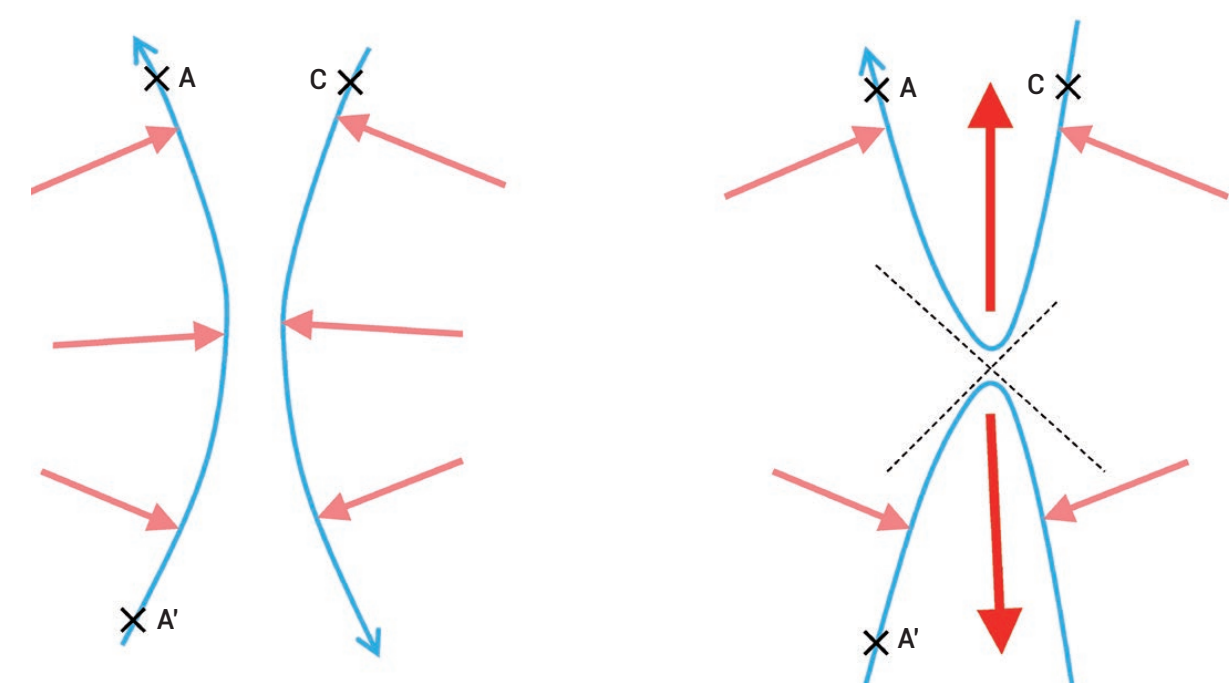

2. Schéma de principe de la reconnexion. Les lignes de champ (en bleu) se rapprochent du point $X$ (en pointillés sur la figure de droite), poussées par le plasma dont la vitesse est tracée en rose. S'il y a reconnexion, le point $A$, initialement connecté magnétiquement au point $A^{\prime}$ (à gauche), peut ainsi se retrouver soudainement connecté au point $\mathrm{C}$ (à droite). Pendant ce phénomène, le plasma se trouve fortement accéléré par la force de tension des lignes de champ (flèches rouges).

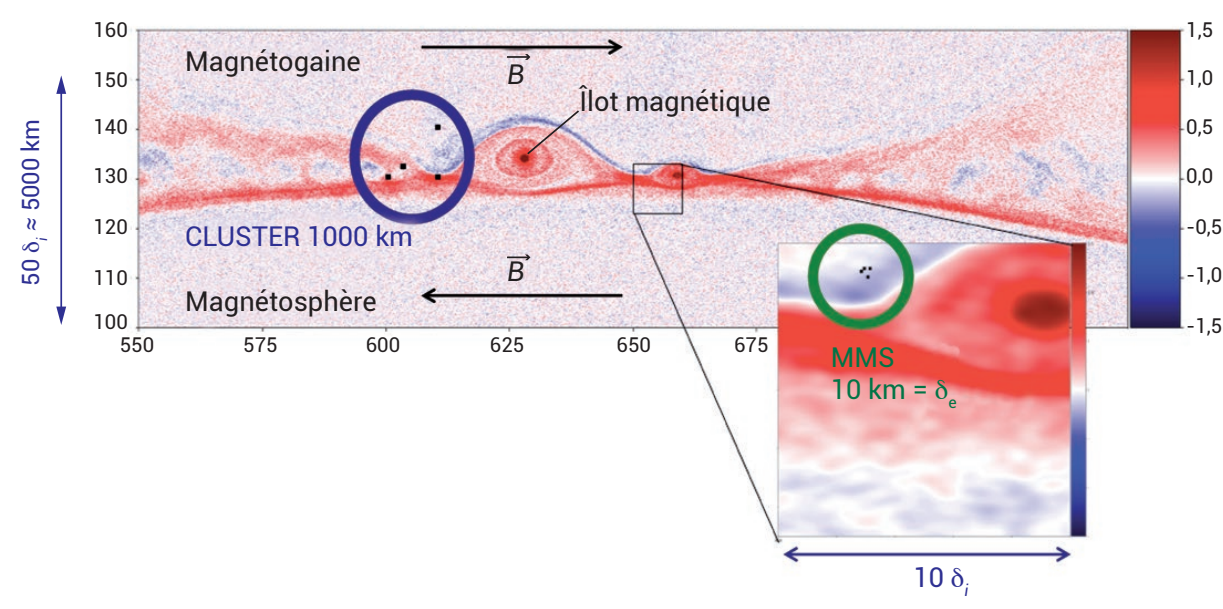

3. Simulation numérique de la magnétopause avec un code PIC (Particle In Cell) 2D. La magnétosphère est en bas (B dirigé vers la gauche), la magnétogaine en haut (B dirigé vers la droite). Les échelles sont indiquées en longueur d'inertie des ions. La composante $\mathrm{j}_{z}$ (perpendiculaire au plan) du courant électrique est représentée en couleur (code couleur à droite de la figure). La magnétopause correspond au maximum de ce courant. On observe l'apparition d'un îlot magnétique dû à la reconnexion. Les quatre points noirs, entourés d'un cercle bleu ou vert donnent I'ordre de grandeur des distances intersatellites, respectivement pour Cluster et pour MMS.

\section{\〉}

parfois, de façon simplifiée, que les lignes de champ se " coupent " et se raccordent différemment. Une ligne du vent solaire peut ainsi se trouver subitement connectée à une ligne liée à la Terre, reliant l'intérieur et l'extérieur de la magnétopause. En conséquence, les particules du vent solaire peuvent pénétrer dans la magnétosphère. Tous les phénomènes magnétosphériques, comme les aurores polaires, sont des conséquences plus ou moins directes de ces reconnexions. Personne, jusqu'ici, n'avait $\mathrm{pu}$ observer en détail le voisinage du " point en X ", l'endroit où se produit la « déconnexion-reconnexion » des lignes de champ. Compte tenu de la nécessité de prendre des mesures aux échelles électroniques, spatiales et temporelles dans ce voisinage, pour comprendre la physique de la reconnexion, on se rend compte de l'intérêt de MMS : les satellites de Cluster étaient trop éloignés (fig. 3) et la résolution temporelle de ses instruments de mesure des particules insuffisante pour atteindre ces échelles.

\section{Les innovations apportées par MMS}

Même si MMS ressemble à Cluster (embarquant des instruments qui mesurent toutes les propriétés du plasma), cette nouvelle mission apporte des progrès très significatifs. La première différence est illustrée sur la figure 3 : les satellites sont souvent plus proches, à des distances de l'ordre de la dizaine de kilomètres. La deuxième innovation est dans les capacités des instruments embarqués. Jusqu'à présent, on détectait les particules à bord des satellites grâce à un spectromètre de masse capturant celles-ci dans un plan. Le satellite tournant sur lui-même, on obtenait une mesure tridimensionnelle de la fonction de distribution à chaque période de rotation du satellite. La résolution temporelle ne pouvait pas être meilleure que la période de rotation (4 s sur Cluster), ce qui se traduisait en particulier par une mesure très imprécise de la vitesse fluide dès que le milieu n'était pas strictement stationnaire sur cette période. Sur MMS, l'instrument FPI est doté de quatre couples de détecteurs. Chaque couple observe un quart du champ de vue grâce à un système de déflecteurs. On effectue ainsi 32 mesures simultanées (fig. 4). On a de cette façon une résolution temporelle inégalée : $0,03 \mathrm{~s}$ pour les électrons, $0,15 \mathrm{~s}$ pour les ions. 
L'IRAP (Institut de Recherche en Astrophysique et en Planétologie) a fourni les 32 galettes à microcanaux qui servent de détecteurs à l'intérieur des analyseurs d'ions. Cette innovation permet, pour la première fois, d'utiliser les mesures de vitesses des ions et des électrons pour calculer la densité de courant, chose qui n'avait jamais pu être faite jusqu'à présent. Sur Cluster on ne pouvait utiliser que le calcul dérivé de l'équation de MaxwellAmpère, en estimant le rotationnel du champ magnétique à partir des mesures en quatre points. La figure 5 montre que les deux calculs donnent sur MMS des résultats tout à fait comparables et se valident l'un l'autre.

En ce qui concerne les champs, on mesure les trois composantes du champ magnétique et du champ électrique (contre deux seulement sur Cluster), en continu et en alternatif. Pour les magnétomètres alternatifs fournis par le LPP, les innovations sont moins dans la sensibilité et la résolution temporelle qui sont semblables à celles de Cluster, que dans l'encombrement et le poids de l'ensemble capteur-préamplificateur. L'optimisation de la forme du noyau magnétique (en donnant à ses extrémités une forme conique) a permis de diminuer l'encombrement des antennes en gardant une bonne sensibilité $\left(0,05 \mathrm{pT} /\left(\mathrm{H}_{\mathrm{z}}\right)^{1 / 2}\right.$ à $\left.1 \mathrm{kHz}\right)$. Dans le même temps, l'électronique du préamplificateur a été intégrée en utilisant une technologie tridimensionnelle développée par la société française 3D PLUS, ce qui a permis de réduire sa masse à 37 grammes. Le plus lourd est maintenant ce qu'on ne peut pas réduire : la connectique.

Ces instruments nombreux et performants fournissent des quantités de données gigantesques, trop importantes pour être transmises au sol par l'antenne de télémesure. Dans le mode appelé "burst" où toutes les données sont acquises à la meilleure résolution possible, il suffit de 15 minutes de données pour remplir la bande passante du satellite sur une journée. Il a donc fallu mettre au point une stratégie pour optimiser les données transmises au sol. La méthode retenue est la suivante : toutes les données sont stockées à haute résolution dans la mémoire du satellite pendant une semaine et un logiciel sélectionne les périodes intéressantes. Mais elles sont aussi transmises au sol dans un mode sommaire pour être analysées par le "Scientist In The Loop"

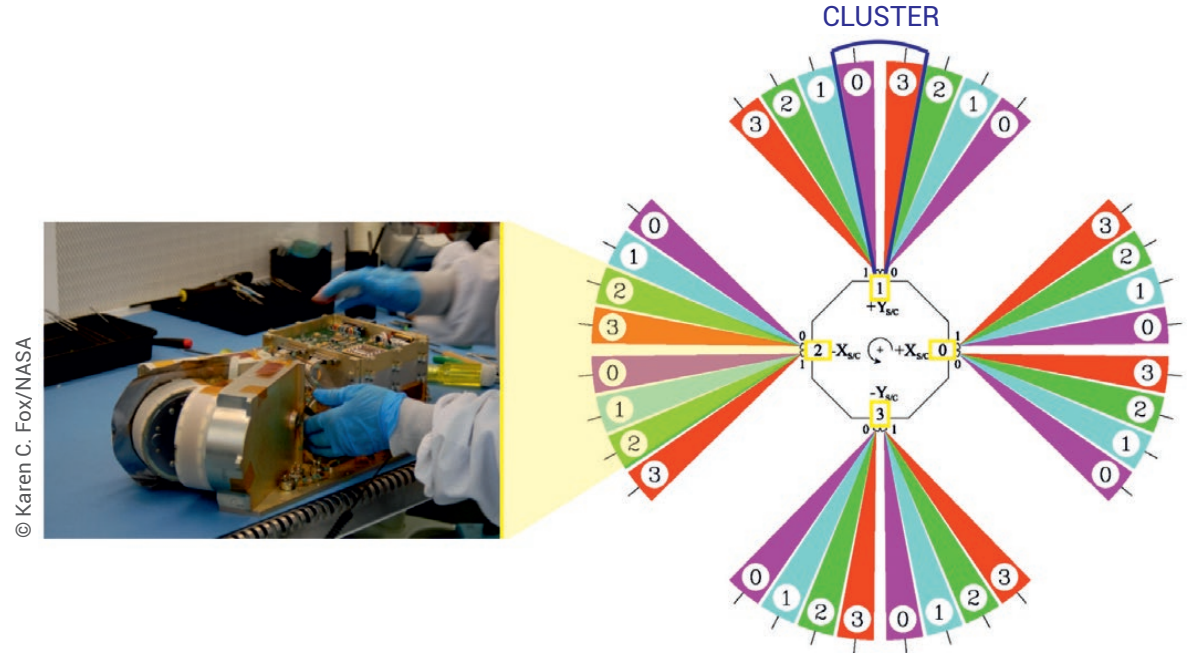

4. Les détecteurs de l'instrument FPI ("Fast Plasma Instrument"). La plate-forme octogonale du satellite est représentée à droite, avec quatre paires de détecteurs de FPI (encadrés de jaune) et leurs champs de vue respectifs en couleur (tiré de Pollock et al., 2016). Pour comparaison, le champ de vue de l'expérience comparable sur Cluster est indiqué en bleu sur le détecteur 1. La photo de gauche est un zoom sur une paire de détecteurs.

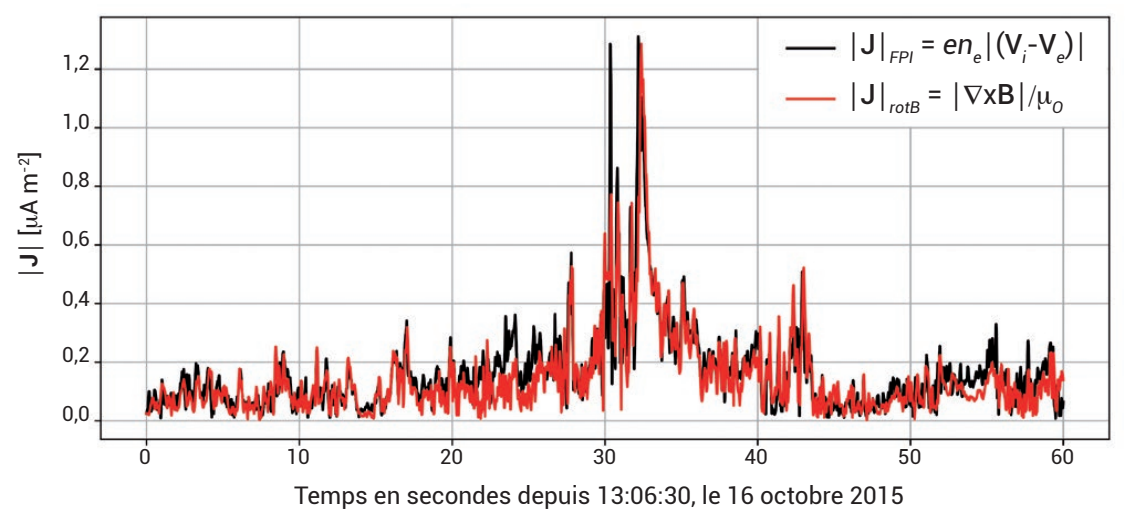

5. Calcul de la densité de courant à partir de l'équation de Maxwell-Ampère (en rouge) et à partir des mesures des vitesses des particules (en noir). La première méthode ne peut fonctionner qu'avec quatre satellites. La seconde se déduit directement des mesures faites à bord d'un satellite : les quatre satellites voyant sensiblement la même chose, on a donc représenté la moyenne.

(SITL). Celui-ci met des notes à chaque intervalle suivant ce qu'il pense être des événements intéressants. Quand il met une note très élevée, les données en mode "burst" sont transmises au sol très rapidement. Quand il met une note moins bonne, le système attend avant de transmettre pour voir s'il n'apparait pas un événement plus intéressant qui pourrait remplacer celui-ci. La responsabilité du SITL tourne toutes les semaines entre les équipes qui sont impliquées dans le projet, de manière à ce qu'il n'y ait pas de biais dans la sélection. Cette collaboration entre l'homme et l'ordinateur fonctionne à merveille et permet de ne rien rater : les événements de reconnexion sont imprévisibles et irréguliers, il peut se passer beaucoup de choses un jour, puis rien le lendemain. De cette façon, on peut transmettre au sol toutes les données nécessaires quand on observe un cas intéressant. 

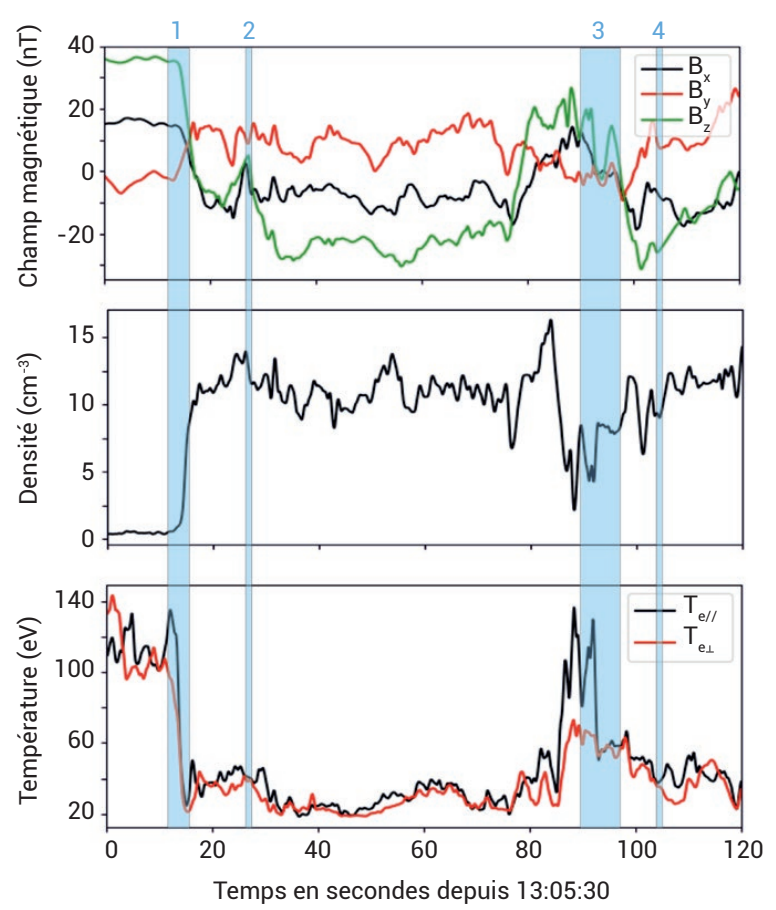

6. Données du satellite MMS1 pour le 16 octobre 2015, à partir de 13:05:30, tracées en fonction du temps : champ magnétique, densité des ions, température des électrons (parallèlement et perpendiculairement au champ magnétique). Les régions identifiées comme clairement à une dimension par l'analyse du tenseur $\nabla B$ sont surlignées en bleu et numérotées de 1 à 4.

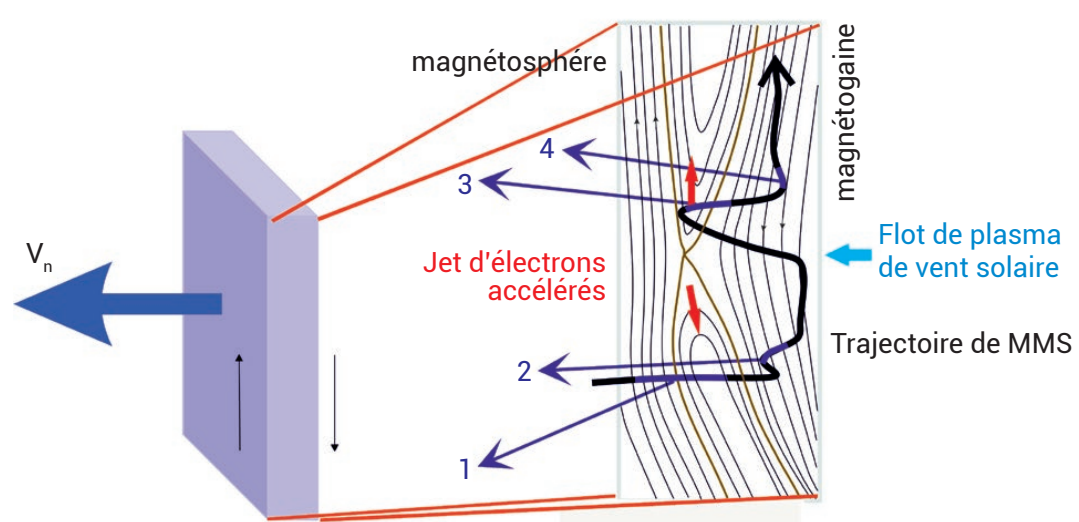

7. Traversée de la magnétopause par MMS : interprétation de la figure 6 (vue d'artiste).

À droite : trajectoire relative des satellites par rapport à la frontière supposée stationnaire pendant le temps de la traversée (dans la réalité c'est plutôt la frontière qui bouge, le mouvement des satellites sur leur orbite, de l'ordre de $5 \mathrm{~km} / \mathrm{s}$, est nettement plus lent que celui de la frontière, de l'ordre de $50 \mathrm{~km} / \mathrm{s}$ ). Les quatre normales représentées correspondent aux zones 1D surlignées en bleu sur la figure 6. La partie gauche représente le modèle simpliste auquel on était limité avant MMS $\left(V_{n}\right.$ est une vitesse normale globale). Le fond de la partie droite de la figure est une carte de ce que pourrait être le champ magnétique à l'intérieur de la frontière, compte tenu des nouvelles informations accessibles.

\section{Références}

G. Belmont et al., Collisionless Plasmas in Astrophysics, Wiley (2013).

G. Belmont, F. Sahraoui et L. Rezeau, "Turbulence dans les plasmas spatiaux", Images de la physique (2007) 29-34.

O. Le Contel et al., Geophys. Res. Lett., 43 (2016) 5943-5952.

\section{\〉> \\ Les premières observations}

La figure 6 montre un exemple de traversée de la magnétopause (de la magnétosphère vers la magnétogaine), observée par l'un des satellites de MMS et identifiée grâce aux modifications importantes de tous les paramètres visibles au début de la période (à partir de $t=14 \mathrm{~s}$ ) : le champ magnétique se retourne, la densité du plasma augmente fortement pendant que la température des électrons chute. Les autres satellites voient des signatures très semblables, les décalages étant difficiles à percevoir ici. Néanmoins, l'utilisation de plusieurs satellites permet d'analyser la structure de la frontière et de vérifier si elle ressemble à l'image simple qu'on se fait d'une discontinuité, frontière plane (donc à une dimension) en mouvement relatif par rapport au satellite (partie gauche de la figure 7). Dans le cas présent, le mouvement apparent du satellite dans la frontière (c'est en réalité la frontière qui se déplace) commence par une traversée franche de la couche de courant de la magnétosphère vers la magnétogaine, qui apparait bien être une discontinuité plane. Ensuite, le satellite reste plus ou moins dans la magnétopause et revient même en arrière pour se retrouver à nouveau dans une région à une dimension juste avant $t=27 \mathrm{~s}$ puis, de nouveau, vers $t=90 \mathrm{~s}$ et enfin, très brièvement, vers $t=103 \mathrm{~s}$. Entre ces régions, qui peuvent être identifiées comme 1D par l'analyse du tenseur $\nabla \mathbf{B}$, on a des régions où le problème est plus complexe (souvent 2D, parfois complètement 3D). Vers $t=85-95 \mathrm{~s}$, on observe sur la température des électrons une forte anisotropie qui correspond à une déformation de la fonction de distribution des électrons associée à un champ électrique parallèle au champ magnétique. La partie droite de la figure 7 illustre comment on peut interpréter la structure de la frontière pendant que les satellites MMS la traversent. La fin de la période correspond très probablement au passage près d'un point en $\mathrm{X}$.

Un deuxième exemple des possibilités offertes par la mission MMS est illustré par la figure 8. On observe sur la figure 8a la signature d'ondes de fréquence de l'ordre de $100 \mathrm{~Hz}$, à des périodes bien localisées dans le temps (zones encadrées en jaune). On constate (fig. 8b) que ces périodes correspondent à un maximum local de la densité du plasma, associé à un minimum du module du champ (zones encadrées en 
marron). Ces structures sont appelées modes " miroir" puisque les électrons peuvent se retrouver piégés à l'intérieur, comme entre deux miroirs magnétiques. Ce qui est nouveau, c'est la possibilité d'obtenir les fonctions de distribution détaillées des électrons à ces échelles de temps. On voit clairement grâce à cela (fig. 8c) que les électrons sont effectivement piégés par le champ magnétique dans les modes miroir (les traits noirs figurent les angles d'attaque entre lesquels les électrons sont piégés).

Le dernier exemple met en lumière le fait que la dynamique de la reconnexion ne peut être traitée sans décrire la dynamique propre des électrons. L'équation fluide de conservation de la quantité de mouvement électronique peut s'écrire sous la forme suivante (loi d'Ohm généralisée), où $\mathrm{P}_{\mathrm{e}}$ est le tenseur de pression électronique, $\mathbf{u}_{\mathrm{e}}$ la vitesse des électrons :

$\mathbf{E}+\left(\mathbf{u}_{\mathrm{e}} \times \mathbf{B}\right)=(\mathbf{j} / \sigma)-\left(\nabla \cdot \mathbf{P}_{\mathrm{e}} / n e\right)-\left(m_{e} / e\right) d_{t} \mathbf{u}_{\mathrm{e}}$ Le premier terme dans le membre de droite $(j / \sigma)$ est le terme classique de résistivité. Il est complètement négligeable ici, car il n'y a pas de friction entre les électrons et les ions : le plasma est « sans collisions ». Quand les autres termes sont négligeables aussi devant $\mathbf{u}_{\mathrm{e}} \times \mathbf{B}$, ce qui est le cas aux grandes échelles, le champ magnétique est gelé dans le fluide électronique. Ces termes deviennent non négligeables pour les échelles plus petites, respectivement, que le rayon de Larmor et la longueur d'inertie des électrons. La qualité des données de MMS permet de mesurer chacun de ces termes séparément. Le gradient du tenseur de pression électronique est calculé avec la même méthode que le rotationnel du champ magnétique, en utilisant les mesures aux quatre points des quatre satellites. On trace ces termes lors d'une traversée de la magnétopause (fig. 9), identifiée grâce au champ magnétique et à la densité (panneaux $a$ et $b$ ). Les différents termes de la loi d'Ohm sont tracés sur le panneau $d$. On constate que les deux derniers termes sont négligeables, sauf dans la partie entourée en rouge : cette zone correspond à la partie de la traversée de la magnétopause où l'on voit des électrons accélérés (panneau c). Le terme d'inertie des électrons (en vert) est ici toujours négligeable. On voit qu'à l'endroit où se produit la reconnexion, on est bien en présence de deux des signatures attendues: électrons accélérés et phénomènes non idéaux (tenseur de pression électronique dominant dans le champ électrique).
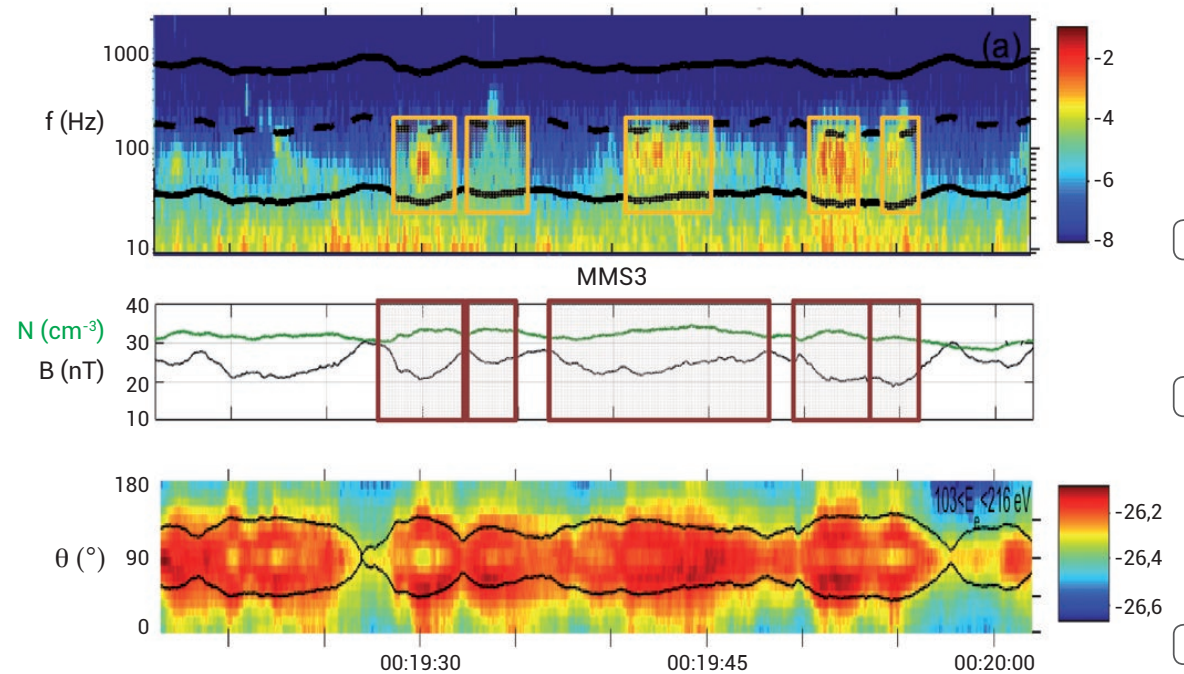

8. Observations d'ondes dans la magnétogaine, le 22 janvier 2016, par le satellite MMS3. En haut : spectrogramme de la composante magnétique des ondes mesurée avec les magnétomètres du LPP (I'amplitude est tracée en code couleur en fonction du temps et de la fréquence). En dessous : module du champ magnétique $\mathbf{B}$ et densité du plasma $\mathrm{N}$ en fonction du temps. Tout en bas : fonction de distribution des électrons tracée en fonction du temps et de l'angle d'attaque $\theta$ (angle de la vitesse des particules avec le champ magnétique statique). Lignes noires : les limites de piégeage des électrons dans le champ magnétique (on reconnait l'allure du module du champ représenté sur la figure b).



9. Mesure par MMS de différents paramètres physiques lors d'une traversée de la magnétopause. Données de MMS1 pour le 27 janvier 2017, autour de 12:05:43, tracées en fonction du temps champ magnétique (a), densité du plasma (b), vitesse des électrons (c), et différents termes de la loi d'Ohm avec les codes couleur indiqués sur la figure (d).

\section{Conclusion}

La reconnexion est un phénomène complexe qui associe grandes et petites échelles, temps longs et temps courts. Les conséquences à grande échelle d'un événement sont la reconfiguration du champ magnétique et une accélération du plasma. Elles sont relativement faciles à observer, car elles durent assez longtemps. Au contraire, les causes sont des phénomènes à petite échelle et de courte durée. Pour les observer, il faut avoir un outil adapté, qui se situe au bon endroit et qui mesure tous les paramètres avec une grande cadence d'échantillonnage au bon moment. On voit qu'avec MMS on s'est donné les moyens de réaliser ces mesures.

Les auteurs remercient Olivier Le Contel, Hugo Breuillard, Giulia Cozzani, Jérémy Dargent et Roberto Manuzzo, ainsi que le CNES et le CNRS qui financent la participation française à MMS (Service national d'observation de l'INSU). 